\title{
Erratum to: Application on Cold Chain Logistics Routing Optimization Based on Improved Genetic Algorithm
}

\author{
Liyi Zhang ${ }^{a}$, Yang Gao ${ }^{b}$, Yunshan Sun ${ }^{a, *}$, Teng Fei ${ }^{a}$, and Yujing Wang ${ }^{b}$ \\ ${ }^{a}$ Information Engineering College, Tianjin University of Commerce, Tianjin, 300134 P.R. China \\ ${ }^{b}$ Economic College, Tianjin University of Commerce, Tianjin, 300134 P.R. China \\ *e-mail: sunyunshan@tjcu.edu.cn \\ Submitted November 14, 2019; accepted for publication November 14, 2019
}

DOI: $10.3103 / \mathrm{S} 0146411619060105$

Page 169, the affiliation under indication $a$ should read as follows:

${ }^{a}$ Information Engineering College, Tianjin University of Commerce, Tianjin, 300134 P.R. China The original article can be found online at https://doi.org/10.3103/S0146411619020032 